\title{
PSYCHOPATHOLOGY AND QUALITY OF LIFE OF MEDICAL STUDENTS AND DOCTORS FROM BRAZIL PARTICIPATING IN A CONTINUING MEDICAL EDUCATION EVENT
}

Arthur Hirschfeld Danila1, Eduardo de Castro Humes ${ }^{2}$

${ }^{1}$ Office of Student Affairs, Faculdade de Medicina, Universidade de Sao Paulo, São Paulo, Brazil.

${ }^{2}$ Students Mental Helath Clinic, Faculdade de Medicina, Universidade de Sao Paulo, São Paulo, Brazil.

${ }^{3} \mathrm{Head}$ of the Psychiatric Outpatient Unit, Hospital Universitario, Universidade de Sao Paulo, São Paulo, Brazil. Keywords: Medical students, Physician mental health, Depression, Anxiety, Quality of Life

Background: Recent systematic reviews with meta-analysis of the Brazilian and international literature present evidence that medical students experience high rates of depressive symptoms and suicidal ideation. Similar findings were observed among resident physicians. The deterioration of mental health in medical students and residents is associated with worse quality of life indicators, defined as the "individual's perception of their position in life in the context of the culture and value systems in which they live and in relation to their goals, expectations, standards and concerns" (World Health Organization). There is also literature that associates psychiatric morbidity in different studies with: living alone and away from family, living away from college, difficulty making friends, sleep problems, poor self-rated academic performance, female gender and be part of a minority, despite low replication. Several studies correlate medical education and practice with the occurrence of mental disorders and reduction in quality of life, less effective patient care, increased medical errors and greater abstention.

Objectives: To evaluate mental health and quality of life of medical students and physicians of the Brazilian state of Tocantins (located in the Northern area of Brazil) participating in a continuing medical education event promoted by the state's Regional Medical Council.

Materials and Methods: In 2018, 49 participants voluntary and anonymously were online surveyed about demographics characteristics, psychopathology measured by the State-Trait Anxiety Inventory and 16-item Quick Inventory of Depressive Symptomatology Self-Report, and quality of life measured by the World Health Organization Quality of Life-Bref (WHOQOL-BREF). The FMUSP Ethical Review Board approved this study (Registration number 01000318.7.0000.0065). All data are presented as mean \pm SD and range for continuous variables and $n$ and percentages (\%) for categorical variables. Mann-Whitney test was conducted to analyze the relationship between gender and continuous variables. We used Spearman's $\rho$ correlation to investigate the relationship between two continuous variables. In all data analysis we used SPSS Statistics for Machintosh (IBM Corp. Released 2016. IBM SPSS Statistics for Macintosh, Version 24.0. Armonk, NY: IBM Corp.). Significance level was set at 0.05 and all tests were bicaudal.

Results and Conclusions: The characteristics of our sample are presented in table 1 and QIDS-SR 16 distribution is presented in figure 1 , State-Anxiety in figure 2 and Trait-Anxiety in figure 3 . We found a high prevalence of psychopathology and lower quality of life among medical residents/physicians attending the event, with similar rates when compared to other studies worldwide.

Female participants presented higher trait anxiety (mean: 45.68 males, 47.57 females, $p=.045$ ), with no statistically significant difference was present for depressive symptoms and state anxiety.
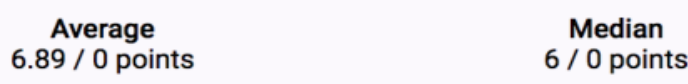

Range
$0-15$ points
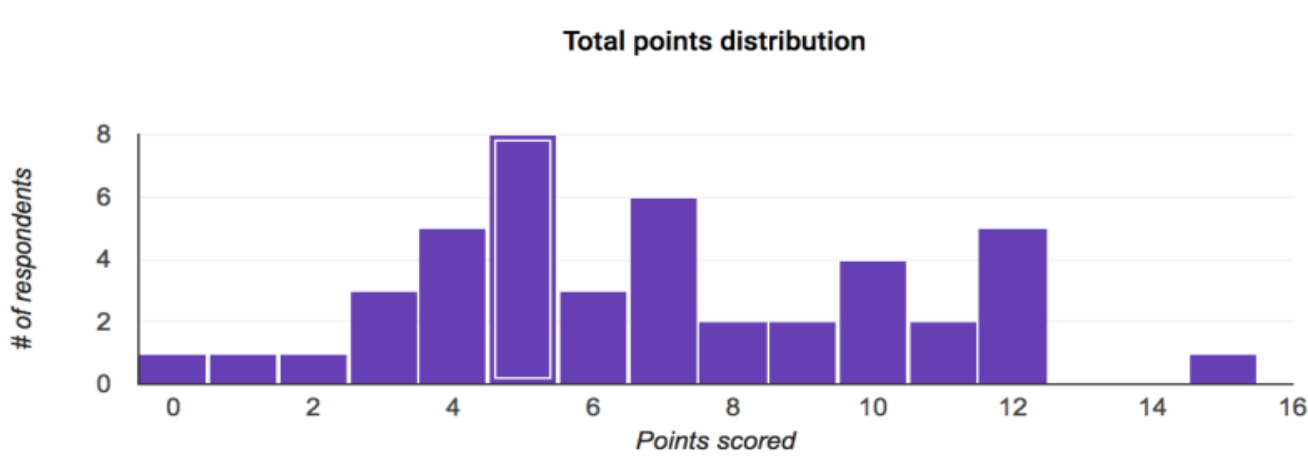

Figure 1: QIDS-SR16 scores
Depressive symptoms, Trait-anxiety and State-anxiety were negatively associated with general scores of quality of life (-.654, $\mathrm{p}<.001 ;-.344, \mathrm{p}=.016 ;-.582, \mathrm{p}<.001)$, psychological quality of life $(-.596, p<.001 ;-.392, p=.005 ;-.529, p<.001)$, social relationships $(-.624, \mathrm{p}<.001 ;-.479, \mathrm{p}<.001 ;-.528, \mathrm{p}<.001)$.

Depressive symptomatology and State-Anxiety were also negatively associated with physical quality of life $(-.471, p=0.001 ;-.409, p=0.004)$ and environment $(-.451, \mathrm{p}=0.001 ;-.453, \mathrm{p}=0.001)$.

This finding can guide future research on interventions to improve mental health amongst Brazilian medical students and professionals.

\begin{tabular}{|l|c|c|c|}
\hline Discrete variables & $\mathbf{n}$ & & (\%) \\
\hline Gender & 49 & & \\
\hline male (n;\%) & & 19 & 57,4 \\
\hline female (n;\%) & & 30 & 42,6 \\
\hline Continuous variables & & mean & SD \\
\hline Age, years & $47^{*}$ & & \\
\hline - mean (SD) & & 30.45 & \pm 9.53 \\
\hline - range & 49 & $17-59$ & \\
\hline STAI-Trait (20-80) & & 46.84 & \pm 3.22 \\
\hline - mean (SD) & & $41-54$ & \\
\hline - range & 49 & & \\
\hline STAI-State (20-80) & & 45.14 & \pm 10.83 \\
\hline - mean (SD) & & $23-63$ & \\
\hline - range & 49 & & \\
\hline QIDS-SR16 (0-27) & & 6.9 & \pm 4.23 \\
\hline - mean (SD) & & $0-23$ & \\
\hline - range & 49 & & \\
\hline $\begin{array}{l}\text { WHOQOL-BREF - } \\
\text { General quality of life (0-100) }\end{array}$ & & 65.98 & \pm 8.91 \\
\hline - mean (SD) & & $44.62-81.54$ & \\
\hline - range & 49 & & \\
\hline $\begin{array}{l}\text { WHOQOL-BREF - } \\
\text { Physical health (0-100) }\end{array}$ & & 61.4 & \pm 8.67 \\
\hline - mean (SD) & & $40-77.14$ & \\
\hline - range & 49 & & \\
\hline $\begin{array}{l}\text { WHOQOL-BREF - } \\
\text { Psychological (0-100) }\end{array}$ & & 65.71 & \pm 10.32 \\
\hline - mean (SD) & & $44.33-86.67$ & \\
\hline - range & & & \\
\hline $\begin{array}{l}\text { WHOQOL-BREF - } \\
\text { Social relationships (0-100) }\end{array}$ & & 67.21 & \pm 18.85 \\
\hline - mean (SD) & & $37.5-87.5$ & \\
\hline - range & & $20-100$ & \\
\hline $\begin{array}{l}\text { WHOQOL-BREF - } \\
\text { Environment (0-100) }\end{array}$ & & & \\
\hline - mean (SD) & & & \\
\hline - range & & & \\
\hline
\end{tabular}

Table 1: Characteristics of participants included in the study STAI: State-Trait Anxiety Inventory; QIDS-SR 16: 16-item Quick Inventory of Depressive Symptomatology Self-Report; WHOQOLBREF: WHO Quality of Life-BREF; SD: standard deviation; total sample $=49$ applicants; ${ }^{*} \mathrm{n}$ may vary as a result of missing data

References:

Pacheco JP, Giacomin HT, Tam WW, Ribeiro TB, Arab C, Bezerra IM, et al. Mental health problems among medical students in Brazil: a systematic review and meta-analysis. Rev Bras Psiquiatr. 2017:0. Puthran R, Zhang MW, Tam WW, Ho RC. Prevalence of depression amongst medical students: a metaanalysis. Med Educ 2016.50(4):456-68.

analysis. Med Educ. 201650(4): Ho 68 . Prevalon Students: A Systematic Review and Meta-Analysis. Jama. 2016;316(21):2214-36.

Mata DA te al. Prevalence of Depression and Depressive Symptoms Among Resident Physicians: A Systematic Review and Meta-analysis. Jama. 2015:314(22):2373-83

World Health Organization. The World Health Organization Ouality of Life Assessment (WHOQOL): development and general psychometric properties. Soc Sci Med. 1998 Jun:46(12):1569-85.

Segal SS, Giordani B, Gillum LH, Johnson N. The Academic Support Program at the University of Michigan School of Medicine. Acad Med. 1999 Apr:74(4):383-5.

Dybye LN, Thomas MR, Shanaft TD. S4stematic revi

Dybyession, anxiety, and other indicators of Wright AA, Katz IT. Beyol

J Med. 2018 Jan 25:378(4):309-311.

Dyrbye LN. Burnout among health care professionals: A

World Heath Organization. WHOQOL-BREF intoduct

Torsesment.

Spielberg $\mathrm{CD}$

Porsuch RL,

Push AJ Trivedi MH, Ibrahim

, Carmody TJ, Arnow B, Klein DN, et al

(O) 